\title{
Transcultural translation and validation of Fugl-Meyer assessment to Italian
}

F. Cecchi, C. Carrabba, F. Bertolucci, C. Castagnoli, C. Falsini, B. Gnetti, I. Hochleitner, G. Lucidi, M. Martini, I. E. Mosca, S. Pancani, A. Paperini, S. Verdesca, C. Macchi \& M. Alt Murphy

To cite this article: F. Cecchi, C. Carrabba, F. Bertolucci, C. Castagnoli, C. Falsini, B. Gnetti, I. Hochleitner, G. Lucidi, M. Martini, I. E. Mosca, S. Pancani, A. Paperini, S. Verdesca, C. Macchi \& M. Alt Murphy (2020): Transcultural translation and validation of Fugl-Meyer assessment to Italian, Disability and Rehabilitation

To link to this article: https://doi.org/10.1080/09638288.2020.1746844

View supplementary material $\widetilde{ }$

曲 Published online: 01 May 2020.

Submit your article to this journal $\longleftarrow$

Џ Article views: 1

Q View related articles $₫$

View Crossmark data $\asymp$ 


\title{
Transcultural translation and validation of Fugl-Meyer assessment to Italian
}

\author{
F. Cecchi ${ }^{a}$, C. Carrabba ${ }^{a}$, F. Bertolucci ${ }^{a}$, C. Castagnoli ${ }^{a}$, C. Falsini ${ }^{a}$, B. Gnetti ${ }^{a}$, I. Hochleitner ${ }^{a}$, G. Lucidi ${ }^{a}$, M. Martini ${ }^{a}$, \\ I. E. Mosca ${ }^{a}$, S. Pancani ${ }^{a}$, A. Paperini ${ }^{a}$, S. Verdesca ${ }^{a}$, C. Macchi ${ }^{a}$ and M. Alt Murphy ${ }^{b}$ (D)

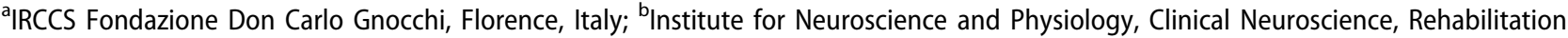 \\ Medicine, Sahlgrenska Academy, University of Gothenburg, Gothenburg, Sweden
}

\section{ABSTRACT}

Purpose: The Fugl-Meyer Assessment is the most used and highly recommended clinical assessment of sensorimotor function after stroke. A standardized use of the scale in different countries requires translation and cultural validation to the target language. The objective of the study was to develop an official Italian version of the scale by transcultural translation and validation.

Methods: A standardized multistep translation protocol was adopted to achieve optimal conceptual and semantic equivalence. The developed Italian version was validated in 10 post-stroke hemiparetic patients. Items with low intra- and interrater agreement, quantified as percentage of agreement $<70 \%$ and/or statistically significant disagreement in relative position or concentration, between different raters were identified and revised.

Results: All motor items received a high level of agreement with values well above $70 \%$. Disagreements were observed in 6 items in the sensory, joint range and pain domains and 1 in one reflex item. Items showing disagreements were discussed and revised to establish the final Italian version.

Conclusions: The culturally validated Italian Fugl-Meyer Assessment can reliably be used in research and in clinical practice. A standardized use will improve the quality of sensorimotor assessment in stroke across Italy and allow reliable comparisons of stroke populations internationally.

IMPLICATIONS FOR REHABILITATION

- The Fugl-Meyer Assessment is the gold standard for evaluation of sensorimotor impairment after stroke.

- Having access to a transculturally validated official Italian version of Fugl-Meyer Assessment will improve the quality of sensorimotor assessment after stroke among Italian health professionals and researchers.

A wider standardized use of the Fugl-Meyer Assessment in Italy will allow reliable international comparison of stroke rehabilitation outcomes.
ARTICLE HISTORY

Received 17 September 2019

Revised 19 March 2020

Accepted 20 March 2020

\section{KEYWORDS}

Fugl-Meyer Scale; Patient

Outcome Assessment

Stroke; Cross-cultural

Comparison; Upper

Extremity; Lower Extremity

\section{Introduction}

Stroke is a leading cause of death and disability worldwide and the motor impairment following stroke is recognized as the main cause of post-stroke disability [1]. Hemiparesis or hemiplegia is the most frequent motor deficit following stroke which significantly affects the activities of daily life, postural control, walking and mobility of the patient [2]. In Italy every year there are about 73000 new hospitalizations due to stroke, about a third of those affected do not survive a year after the event, while another third survives with a significant disability [3]. The number of people currently living in Italy with the consequences of stroke is estimated approximately to 350 000. Stroke costs more than 3000 million euros to the National Health System, and 53 per capita [3].

The evaluation of motor function is necessary to understand the mechanisms of motor control and learning, which are the key elements of the post stroke rehabilitation. The possibility of monitoring patient's progress by using a quantitative and qualitative assessment of motor and sensory deficits allows the rehabilitation team to define specific and personalized rehabilitation goals in order optimize treatment selection and functional recovery. Many clinical tools are available for evaluation of functional disabilities and residual motor function in hemiplegic patients [4,5]. However, it is extremely difficult to measure sensorimotor recovery from stroke, because of the heterogeneity of this conditions and the high individual variability in spontaneous recovery. The more general scales, such as, Modified Ranking Scale and the National Institutes of Health Stroke Scale may be inadequate to capture the dynamic process of motor recovery after stroke [6]. On the other hand, the Fugl-Meyer Assessment (FMA), originally published both in English and Swedish by Fugl-Meyer et al. 1975, being the first quantitative standardized assessment tool for the sensorimotor impairment after stroke [7], has been shown to accurately describe sensorimotor impairment after stoke and to be sensitive to changes over time or in response to specific treatment. Indeed, extensive literature has shown that FMA is easy to use and has excellent validity, reliability and responsiveness $[4,8-10]$. The FMA is still recognized as the gold standard for clinical use and research studies worldwide $[11,12]$. 
The systematic use of FMA is strongly recommended both for research purposes and for clinical practice $[4,13]$. The FMA is currently the only impairment level tool recommended to be included in every stroke recovery trial [12]. To meet these recommendations there is an urgent need to ensure that available translations of the FMA in different countries are consistent with the original version and feasible in the target setting. There is also a need to evaluate the developed versions of the scale in different languages regarding reliability in order to ensure a consistent use of the scale.

An official translation of the original FMA is available in several languages for non-profit clinical and research use (www.neurophys.gu.se/rehabmed) $[14,15]$. Although the FMA has been extensively used in the Italian context [16-20], no official FMA version translated and validated to Italian is currently available.

The importance of adapting instruments to current research settings is universally acknowledged and required in order to ensure that concepts explored by a tool are equal between the original and target language, time and context [21]. A comprehensive linguistic translation process and a cross-cultural adaption process are both necessary to ensure construct validity and reliability of the adapted instrument [22]. Finally, the evaluation of the operational equivalence of the translated tool, meaning that similar format, instructions, mode of administration and measurement methods can be applied to the target populations, is also necessary to adapt a validated instrument to a different language, setting and time [23].

The aim of this study was to develop a transcultural translation of the FMA in Italian, both for the upper and lower extremity according to a standardized procedure and to validate the official Italian version of the FMA in a group of post-stroke patients.

\section{Materials and methods}

\section{The Fugl-Meyer assessment}

The motor domains of the Fugl-Meyer Assessment of Upper Extremity (FMA-UE) and Lower Extremity (FMA-LE) rely on direct observation of the motor performance at each item using a 3point ordinal scale $(0=$ cannot perform, $1=$ performs partially, and $2=$ performs fully) [7]. FMA-UE includes four motor subscales: A. Upper Extremity (0-36 points), B. Wrist (0-10 points), C. Hand (0-14 points), D. Coordination/Speed (0-6 points). The total maximum score indicating a good function is 66 points. The FMA-LE with total score of 34 points includes two subscales: E. lower Extremity (0-28 points) and F. Coordination/Speed (0-6 points).

Along with the evaluation of the motor function, the sensory function, passive range of motion and pain during passive movement are evaluated by using the same ordinal 3-point scale. The non-motor domains of the FMA are: H. Sensation (anesthesia, hypoesthesia/dysesthesia or normal - 0-12 points), J. Passive Joint Movement (only a few degrees, decreased or normal - 0-24 points), and Pain (pronounced, mild to moderate or absent 0-24 points). These are assessed both in upper and lower limb.

\section{Translation process}

The permission to translate the original FMA to Italian was received from the University of Gothenburg, the holder of the original version of the scale. The whole sensorimotor assessment for both upper and lower limb including the non-motor domains was translated. The methodological procedures for translation and cultural adaptation of the FMA as suggested by Barbosa et al. were employed [14]. A forward and backward translation along with a rigorous multi-step revision, and drafting of the working and final versions was performed to determine the conceptual, semantic and operational equivalence of the translated scale $[14,24,25]$. A flowchart of the multistep process of translation and cultural validation is shown in Figure 1.

A multidisciplinary team from the Stroke Research Group of Fondazione don Carlo Gnocchi Scientific Institute composed the expert group leading the translation processes. A Swedish researcher with extensive experience and knowledge of the original scale was referred to as external expert. The expert group included 3 medical doctors (specialist in rehabilitation medicine), 1 neurologist, 3 physiotherapists, 1 neuropsychologist and 1 bioengineer, all experienced in Stroke rehabilitation and research. All Italian researchers composing the expert group are fluent both in Italian and English language. Further, two official bilingual professional translators (English and Italian) performed the forward and backward translations; both had expertise in medical translations but neither of them was familiar with the original FMA English version and the translator who performed the back translation remained blind to it throughout the translation process.

The FMA-UE, FMA-LE including the non-motor domains were translated to Italian (forward translation), based on the original English version. An official translator (bilingual, native speaker of the target language) performed the first translation. This translation was subsequently revised and compared with the original version by the expert team and the discrepancies in language were discussed until the shared consensus was reached for the drafting of the first translated version in Italian.

The drafted first Italian version was then independently backtranslated to English (backward translation) by a second independent official bilingual translator (native speaker of English and fluent in Italian), to ensure that the content and the translation corresponded to the semantic characteristics of the original version.

The original and the back-translated version of the scale were compared and discussed to draft a version relevant to Italian context corresponding to the original English version in terms of conceptual and cultural equivalence. This resulted in the $2^{\text {nd }}$ revised Italian version that was subsequently used for pilot validation.

\section{Clinical validation}

In order to test the translated Italian version in a clinical stroke sample, a pilot study was conducted in 10 patients with poststroke hemiparesis. The participants were recruited consecutively among post-stroke patients admitted to the Neurological Rehabilitation Facility of Fondazione don Carlo Gnocchi, Scientific Institute, Florence. The inclusion criteria were: stroke-related paresis in upper and/or lower extremity (as assessed and documented in the patient's medical record by a clinical examination performed by a MD specialist either in neurology or in physical and rehabilitation medicine and documented), age 18-95, stroke onset within 3 months. Information on global disability level was collected by the Modified Rankin Scale [26]. The exclusion criteria were: visual and hearing disorders, amputation of a limb (upper or lower), cerebellar stroke, previous stroke, cognitive decline (Mini Mental State Examination <21) [27], or other condition that would limit cooperation in the administration of the FMA scale. All eligible patients were asked to sign a written informed consent to participate in the pilot study. Ethical approval was achieved from the Ethical Committee Area Vasta Centro, Florence, Italy. 
Forward translation from the English version by an official translator

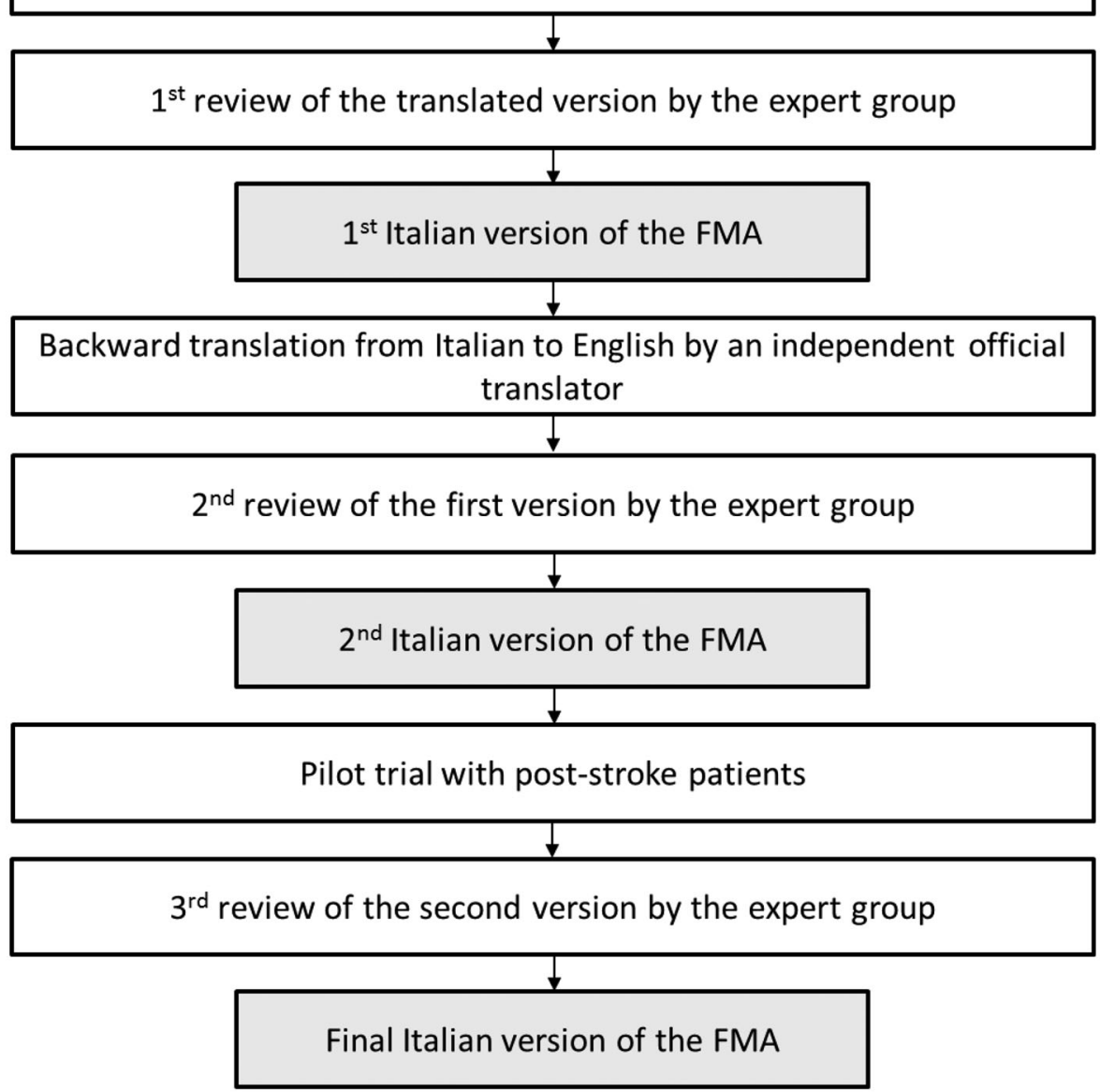

Figure 1. Flowchart over the step-wise translation and cultural adaptation procedure.

An intra- and interrater reliability study design was used to identify items of the scale showing disagreements that could be caused by rater errors, such as administration method, understanding of the scoring scale and patient variance, as well as by language inaccuracies in the translated version among raters. Two licensed physiotherapists, fluent in Italian and conversant with the administration and scoring of the FMA, administered the scale and evaluated the patients' performance during the FMA execution, simultaneously but independently, for two consecutive days. During the first day of evaluation, one of them administered the test by instructing, assisting and scoring the patient's performance and the other assigned an independent score by observing the test administration. (this means that 2 independent scores were obtained from each test occasion and tested for inter-rater reliability). The examiners did not compare the scores during the test session, nor later, and the filled tests remained blind to the other examiner until all assessments were completed.

The rank-based statistical methodology (Svensson method) particularly designed for paired ordinal data (the software is available at http://avdic.se/svenssonsmetod.html) was used for the analysis of inter- and intra-rater reliability of the pilot data [28-30]. The consensus level ( $P A=$ percentage of agreement), between the first and the second observation (for each rater) and between the two different raters (during the same session), was estimated for each individual item of the FMA. According to previous studies, an agreement $\geq 70 \%$ was considered satisfactory and an agreement $\geq 90 \%$ excellent [14]. The systematic disagreement between raters was evaluated by the Relative Position and the Relative Concentration [28,29]. The Relative Position indicates the extent to which the distribution of scores from an assessment is systematically shifted towards higher or lower categories. The Relative Concentration shows whether the scores are more or less concentrated towards the central categories of the scale compared to the other assessment. The Relative Position and the Relative Concentration values can vary from -1 to 1 , where 0 means no difference between raters. Values outside the range between -0.1 and 0.1 were considered as clinically relevant disagreements. The Relative Rank Variation indicates non-systematic disagreement caused by individual variability. A value $<0.1$ means that the difference is negligible. Statistically significant disagreements in Relative Position and the Relative Concentration and Relative Rank Variation were indicated in cases when the 95\% confidence interval that did not include the value zero.

All the disagreements found in the statistical analysis were used as indicators of elements that could have been interpreted differently by two different examiners. These elements were examined to ascertain whether the disagreements they contained could have been caused by translational discrepancies. All the questions and uncertainties highlighted during the trial were reported and reviewed by the expert group, bilingual experts and the external expert to reach a consensus regarding the final version of the FMA. 


\section{Results}

\section{Forward translation}

The first version of the Italian FMA, translated from English, showed some linguistic differences. These differences were addressed during the first review by the expert group and resulted in some contextual changes to this version. The changes consisted mainly of clarifications regarding the manner of expressing concepts. English consists in fact of a concise idiom, characterized by short and slender sentences. Italian, on the other hand, seeks a long-winded style, rich in words, often producing more detailed and elaborated sentences.

The anatomical terms and the descriptions of positions were clearly described and the translation did not require significant changes. Only a few terms required a revision in terms of adaptation to a medical Italian language, such as the term "tendons of hamstrings", which in the literal translation resulted as "tendini del garretto", was modified into "tendini del compartimento posteriore della coscia" ("tendons of the muscles of the rear thigh compartment"); also the term "sensation", which literally translates into "sensazione" (perception, feeling), in the clinical context is more correctly identified with "sensibilità".

The PIP, DIP, MCP and IP acronyms referring to interphalangeal joints and metacarpophalangeal joints have been replaced with IFP, IFD, MCF and IF (indicating the anatomical terms of: interfalangea prossimale, interfalangea distale, metacarpo-falangea e interfalangea).

\section{Backward translation}

Comparing the original English version with the backward translation some discrepancies were found. In the upper extremity motor domain of the hand (C.Hand) it was difficult to find agreement for the translation of "against a tug". The forward translation literally reported "se tirato" (literally "if pulled"), while the multidisciplinary team had agreed on "contro trazione" (literally "against a traction"). In the backward translation, however, the conceptual translation from the Italian led to the English: "if pulled". In the following discussions, it was concluded that this was a non-significant inconsistency which did not affect the administration of the scale. For the final version the "contro trazione" was agreed upon.

The original term "sensation" (sensazione) was back translated into "sensitivity" (sensibilità) which corresponds to the most used clinical term for sensory perception.

The Italian acronyms IFP, IFD, MCF and IF (in Italian respectively for the terms of: interfalangea prossimale, interfalangea distale, metacarpo-falangea e interfalangea) were reversed back to the original PIP, DIP, MCP and IP acronyms referring to the interphalangeal joints and metacarpophalangeal joints.

\section{Clinical validation}

During the study period, lasting 1 month, and completed when the final assessment of the $10^{\text {th }}$ patient was done, 43 patients with a diagnosis of stroke admitted to the rehabilitation department were screened for eligibility within the first week from admission. Of them, 13 patients met the inclusion criteria. Of these, 3 patients were excluded due to clinical complications during hospitalization. Demographic data (age, sex, education), diagnosis of stroke (type, location), Modified Rankin Scale [26] and cognitive status (MMSE) [27] were recorded for each enrolled patient for background information (Table 1).

The level of agreement between and within the assessors was in general high both for the FMA-UE and FMA-LE. Most of the items showed excellent agreement (PA 90-100\%). Apart from two items showing agreement of $60 \%$ (light touch in hand and position sense in thumb), an agreement above $70 \%$ was observed for all items. Systematic statistically significant disagreements (RP or $\mathrm{RC} \geq 0.1$ ) either in position (RP) or concentration (RC) were observed in extensor reflex activity in arm, light touch in hand, passive ankle dorsiflexion, joint pain during passive wrist extension and internal rotation of the hip joint. No individual disagreements, measured as random variance (RV), were observed in any items. The PA values for each item of the FMA are shown in Supplementary Tables 1 and 2.

\section{The final Italian version}

Based on the analysis of disagreements but also on the experiences and perceptions of the raters, the following changes were made to the final Italian version of the FMA.

The expression "voluntary movement with little or no synergy" has been changed from "movimento volontario con scarsa sinergia" to "movimento volontario con sinergia ridotta o assente" ("voluntary movement with reduced or absent synergy"). In the motor domain for the evaluation of the upper extremity, in the Wrist subscale (B), the wording "full active range of motion, smoothly" has been changed from the literal "ROM attivo completo, fluidamente" to "ROM attivo completo e fluido" ("full and fluid active $R O M$ ") and also "complete and smooth circumduction" has been translated to "circonduzione completa e fluida" ("complete and fluid circumduction"). Within the subscale of the Hand (C), an item that required a more careful revision to guarantee a conceptual equivalence as well as allowing a more rapid understanding of the concept was the expression "the objects are interposed"

Table 1. Characteristics of the sample included in the clinical validation.

\begin{tabular}{|c|c|c|c|c|c|c|c|c|c|c|c|}
\hline ID & $\begin{array}{c}\text { Age } \\
\text { (years) }\end{array}$ & Sex & $\begin{array}{l}\text { Education } \\
\text { (years) }\end{array}$ & $\begin{array}{l}\text { Stroke } \\
\text { type }\end{array}$ & $\begin{array}{l}\text { Lesion } \\
\text { side }\end{array}$ & $\begin{array}{l}\mathrm{mRS} \\
(0-5)\end{array}$ & $\begin{array}{l}\text { MMSE } \\
(0-30) \\
\end{array}$ & $\begin{array}{c}\text { FMA-UE } \\
(0-66)\end{array}$ & $\begin{array}{c}\text { FMA-LE } \\
(0-66)\end{array}$ & $\begin{array}{l}\text { Date of } \\
\text { Stroke }\end{array}$ & $\begin{array}{l}\text { Days of ad. } \\
\text { FMA after Stroke }\end{array}$ \\
\hline 1 & 94 & $\mathrm{~F}$ & 5 & Ischemic & $\mathrm{R}$ & 3 & 27 & 23 & 27 & 09/02/2019 & 23 \\
\hline 2 & 75 & $M$ & 5 & Ischemic & $\mathrm{R}$ & 4 & 21 & 0 & 4 & 08/02/2019 & 33 \\
\hline 3 & 74 & $\mathrm{~F}$ & 5 & Hemorrhagic & $\mathrm{L}$ & 3 & 23 & 4 & 11 & 20/02/2019 & 21 \\
\hline 4 & 43 & $M$ & 13 & Ischemic & $\mathrm{L}$ & 1 & 21 & 42 & 33 & 03/03/2019 & 22 \\
\hline 5 & 46 & $M$ & 18 & Ischemic & $\mathrm{R}$ & 0 & 28 & 65 & 34 & $25 / 02 / 2019$ & 21 \\
\hline 6 & 82 & $M$ & 5 & Hemorrhagic & $\mathrm{R}$ & 2 & 30 & 4 & 10 & 01/03/2019 & 27 \\
\hline 7 & 44 & $\mathrm{~F}$ & 8 & Ischemic & $\mathrm{L}$ & 2 & 30 & 4 & 12 & 20/03/2019 & 15 \\
\hline 8 & 57 & $M$ & 13 & Hemorrhagic & $\mathrm{R}$ & 2 & 30 & 54 & 28 & 14/03/2019 & 19 \\
\hline 9 & 61 & $\mathrm{~F}$ & 13 & Ischemic & $\mathrm{R}$ & 2 & 30 & 66 & 34 & $17 / 01 / 2019$ & 24 \\
\hline 10 & 66 & $M$ & 8 & Ischemic & $\mathrm{L}$ & 3 & 27 & 6 & 0 & $01 / 02 / 2019$ & 18 \\
\hline
\end{tabular}

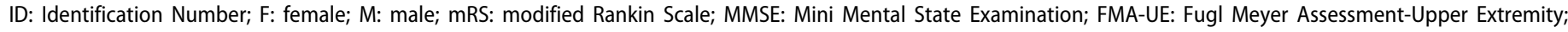
FMA-LE: Fugl Meyer Assessment-Lower Extremity; Date of stroke; Days of administration of Fugl Meyer Assessment after stroke. 
whose literary translation to "gli oggetti sono interposti" ("the objects are interposed"), does not allow to clarify how the therapist administered the objects to the patient. After discussions, the team agreed to the formulation of a more descriptive expression "gli oggetti sono posti vicino alla mano del paziente - il foglio può essere posto tra le dita, se il paziente abduce attivamente il pollice)" ("the objects are placed close to the patient's hand - the piece of paper can be interposed between the patient's fingers if thumb is actively abducted)"), to allow the therapist to have a clearer definition of the modalities of administration of the task.

In the subscale for the evaluation of joint pain we have replaced the term "a little pain" with "dolore lieve-moderato" ("mild to moderate pain"), as it allows us to identify in a linear way the progress of painful symptoms.

The final Italian translation of the original FMA is available for non-profit use at www.neurophys.gu.se/rehabmed.

\section{Discussion}

Clinical outcome measures, such as, questionnaires and clinical scales developed and validated in an original language must be translated and validated to the target language to allow a valid and reliable clinical and scientific use [31]. A prerequisite for the correct use of these assessment tools in research and in clinical practice is obtaining the maximum semantic-conceptual equivalence between the new translated versions and the original version [32]. A rigorous methodological approach following standardized cross-cultural translation and adaptation is essential to ensure that not only linguistic, but also cultural adaptations are taken into account in this process [31]. In this study, we adopted a standardized rigorous process of transcultural translation and validation as previously described by Barbosa et al. [14], to develop an official Italian translation of the original FMA.

Even when the FMA is the most frequently used outcome measure in stroke arm rehabilitation trials, modifications are common $[11,14,33]$. This inconsistency will hamper the possibility to pool and compare data from different studies and synthesize evidence for clinical guidelines. There are many initiatives in Europe and around the world to establish stroke registries to improve the quality of care and rehabilitation. A prerequisite for including an outcome measure to these registries is that the measure is consistently used in clinical settings. The current study will provide an official transculturally validated FMA available for all health care providers in Italy, which will improve the wider consistent use of the scale across the country. The study will hopefully also guide and encourage researches in other parts of the world to establish their official transculturally validated FMA for clinical and research use.

Currently, the original FMA is officially translated to Norwegian, Danish and Spanish-Colombian. In the development of the Colombian Spanish version the rigorous methodology of transcultural translation and validation, as also used in this study, was first reported [14]. Similarly to development of Colombian Spanish version, we used a clinical sample to verify possible inaccuracies in the translated Italian version and to identify problematic areas in practical application. A larger trial on a stratified population of Italian subacute stroke patients will be performed to extend the validation and to verify the responsiveness.

Unlike the study that led to the validation of the FMA in Spanish-Colombian [14], we included patients in the early subacute phase (15-30 days post stroke) rather than 10 day post stroke and the age limit was set to 95 years. These inclusion criteria were considered to match the patient population commonly admitted to rehabilitation units. Indeed, we included a 94 years old patient, who presented a high degree of compliance and a cognitive status largely suitable for the administration of the FMA. In contrast to previous translations we also decided to translate the whole FMA and not only the motor assessment, since both in research and in clinical practice a complete sensorimotor assessment would provide a more comprehensive evaluation of the impairments, as intended by those who developed the original version.

The clinical validation of the Italian FMA version showed that the agreement within and between raters was high for most items and satisfactory for all but two items. The reliability was particularly high for the items that assess the motor performance. In contrast, six items of the sensory, passive joint motion and pain domain and one item in the Upper Extremity reflex activity showed disagreements. These items were carefully revised to confirm that there were no inconsistencies dependent on the description of the items. The intra- and interrater reliability of the nonmotor domains of the FMA has reported to be lower compared to the motor domain $[9,33]$, which needs also to be considered when interpreting the result from the current pilot testing. The clinical validation provided feedback for final adjustments made to the developed official Italian version of the FMA.

One limitation of our study was that the sample size of the pilot validation was small. The final Italian FMA version was tested on 10 stroke patients with hemiparesis, similar to the Columbian study [14]. A larger clinical study is planned to confirm the reliability and to establish the responsiveness of the Italian FMA in a subacute stroke population [15]. Another limitation was that the raters did not underwent a specific joint training of the FMA prior the pilot validation. However, both raters were licensed physiotherapists with good experience of using the FMA scale in patients with stroke. The is also a need to verify the responsiveness of the Italian FMA. These limitations suggest caution when using the Italian version of the FMA in research before a more extensive evaluation of psychometric properties has been done.

\section{Conclusions}

A comprehensive translation process and cross-cultural validation of the FMA showed that the final version of the Italian FMA is valid regarding the language and context. We also verified that agreement between and within raters was good, particularly for the motor items when the Italian FMA was used in a sample of stroke patients. Based on these results we can recommend and advocate the use of the current official Italian FMA translation in research and in clinical practice. The standardized and consistent use of the FMA scale will improve the quality of sensorimotor assessment in stroke rehabilitation and thereby allow reliable comparison of the stroke outcome across regions and countries.

\section{Acknowledgements}

The authors thank all patients who participated to this study.

\section{Disclosure statement}

No potential conflict of interest was reported by the author(s).

\section{Funding}

This study was funded by Ministero della Salute. 


\section{ORCID}

M. Alt Murphy (D) http://orcid.org/0000-0002-3192-7787

\section{References}

[1] Thrift AG, Cadilhac DA, Thayabaranathan T, et al. Global stroke statistics. Int J Stroke. 2014;9(1):6-18.

[2] Mayo NE, Wood-Dauphinee S, Ahmed S, et al. Disablement following stroke. Disabil Rehabil. 1999;21(5-6):258-268.

[3] Stevens EGV, Emmett ES, Wang Y, et al. The Burden of Stroke in Europe: Overview of stroke burden and care in each EU and SAFE member country. Stroke Alliance for Europe; 2017.

[4] Alt Murphy M, Resteghini C, Feys P, et al. An overview of systematic reviews on upper extremity outcome measures after stroke. BMC Neurol. 2015;15:29.

[5] Salter K, Jutai JW, Teasell R, et al. Issues for selection of outcome measures in stroke rehabilitation: ICF Body Functions [Research Support, Non-U.S. Gov't]. Disabil Rehabil. 2005;27(4):191-207.

[6] Brott T, Adams HP, Jr., Olinger CP, et al. Measurements of acute cerebral infarction: a clinical examination scale. Stroke. 1989;20(7):864-870.

[7] Fugl-Meyer AR, Jaasko L, Leyman I, et al. The post-stroke hemiplegic patient. 1. a method for evaluation of physical performance. Scand J Rehabil Med. 1975;7(1):13-31.

[8] See J, Dodakian L, Chou C, et al. A standardized approach to the Fugl-Meyer assessment and its implications for clinical trials. Neurorehabil Neural Repair. 2013;27(8):732-741.

[9] Lin JH, Hsu MJ, Sheu CF, et al. Psychometric comparisons of 4 measures for assessing upper-extremity function in people with stroke. Phys Ther. 2009;89(8):840-850.

[10] Gladstone DJ, Danells CJ, Black SE. The fugl-meyer assessment of motor recovery after stroke: a critical review of its measurement properties [Research Support, Non-U.S. Gov't, Review]. Neurorehabil Neural Repair. 2002;16(3):232-240.

[11] Duncan Millar J, van Wijck F, Pollock A, et al. Outcome measures in post-stroke arm rehabilitation trials: do existing measures capture outcomes that are important to stroke survivors, carers, and clinicians. Clin Rehabil. 2019; 33(4):737-749.

[12] Kwakkel G, Lannin NA, Borschmann K, et al. Standardized measurement of sensorimotor recovery in stroke trials: consensus-based core recommendations from the Stroke Recovery and Rehabilitation Roundtable. Int J Stroke. 2017; 12(5):451-461.

[13] Sullivan JE, Crowner BE, Kluding PM, et al. Outcome measures for individuals with stroke: process and recommendations from the American Physical Therapy Association neurology section task force. Phys Ther. 2013;93(10):1383-1396.

[14] Barbosa NE, Forero SM, Galeano CP, et al. Translation and cultural validation of clinical observational scales - the FuglMeyer assessment for post stroke sensorimotor function in Colombian Spanish. Disabil Rehabil. 2019;41(19):2317-2323.

[15] Hernandez ED, Galeano CP, Barbosa NE, et al. Intra- and inter-rater reliability of Fugl-Meyer Assessment of Upper Extremity in stroke. J Rehabil Med. 2019;51(9):652-659.

[16] Gandolfi M, Vale N, Dimitrova EK, et al. Effectiveness of robot-assisted upper limb training on spasticity, function and muscle activity in chronic stroke patients treated with botulinum toxin: a randomized single-blinded controlled trial. Front Neurol. 2019;10:41.
[17] Kiper P, Szczudlik A, Agostini $M$, et al. Virtual reality for upper limb rehabilitation in subacute and chronic stroke: a randomized controlled trial. Arch Phys Med Rehabil. 2018; 99(5):834-842.

[18] Jonsdottir J, Thorsen R, Aprile I, et al. Arm rehabilitation in post stroke subjects: $A$ randomized controlled trial on the efficacy of myoelectrically driven FES applied in a task-oriented approach. PloS One. 2017;12(12):e0188642.

[19] Germanotta M, Cruciani A, Pecchioli C, et al. Reliability, validity and discriminant ability of the instrumental indices provided by a novel planar robotic device for upper limb rehabilitation. J Neuroeng Rehabil. 2018;15(1):39.

[20] Aprile I, Germanotta M, Cruciani A, et al. Upper Limb Robotic Rehabilitation after Stroke: A Multicenter Randomized Clinical Trial. J Neurol Phys Ther. 2020;44(1):3-14.

[21] Schuster C, Hahn S, Ettlin T. Objectively-assessed outcome measures: a translation and cross-cultural adaptation procedure applied to the Chedoke McMaster Arm and Hand Activity Inventory (CAHAl). BMC Med Res Methodol. 2010;10:106.

[22] Gjersing L, Caplehorn JR, Clausen T. Cross-cultural adaptation of research instruments: language, setting, time and statistical considerations. BMC Med Res Methodol. 2010;10:13.

[23] Reichenheim ME, Moraes CL. [Operationalizing the crosscultural adaptation of epidemiological measurement instruments]. Rev Saude Publica. 2007;41(4):665-673.

[24] Michaelsen SM, Rocha AS, Knabben RJ, et al. Translation, adaptation and inter-rater reliability of the administration manual for the Fugl-Meyer assessment. Rev Bras Fisioter. 2011;15(1):80-88.

[25] World Health Organization. Process of translation and adaptation of instruments. [cited 2020 Mar 25]. Available from: http:// www.who.int/substance_abuse/research_tools/translation/en/

[26] van Swieten JC, Koudstaal PJ, Visser MC, et al. Interobserver agreement for the assessment of handicap in stroke patients. Stroke. 1988;19(5):604-607.

[27] Folstein MF, Folstein SE, McHugh PR. Mini-mental state. A practical method for grading the cognitive state of patients for the clinician. J Psychiatric Res. 1975;12(3):189-198.

[28] Svensson E. Different ranking approaches defining association and agreement measures of paired ordinal data. Stat Med. 2012;31(26):3104-3117.

[29] Svensson E, Holm S. Separation of systematic and random differences in ordinal rating scales. Stat Med. 1994; 13(23-24):2437-2453.

[30] Avdic A, Svensson E. Svenssons method (Version 1.1) Örebro 2010. Interactive software supporting Svenssons method. [cited 2020 Apr 17]. Available from: http://avdic.se/svenssonsmetod.html

[31] Wild D, ISPOR Task Force for Translation and Cultural Adaptation, Grove A, Martin M, et al. Principles of Good Practice for the Translation and Cultural Adaptation Process for Patient-Reported Outcomes (PRO) measures: report of the ISPOR Task Force for Translation and Cultural Adaptation. Value Health. 2005;8(2):94-104.

[32] Sousa VD, Rojjanasrirat W. Translation, adaptation and validation of instruments or scales for use in cross-cultural health care research: a clear and user-friendly guideline. J Eval Clin Pract. 2011;17(2):268-274.

[33] Sullivan KJ, Tilson JK, Cen SY, et al. Fugl-Meyer assessment of sensorimotor function after stroke: standardized training procedure for clinical practice and clinical trials. Stroke. 2011;42(2):427-432. 